\title{
Do language change rates depend on population size?
}

\author{
Søren Wichmann, Dietrich Stauffer, Christian Schulze, Eric W. Holman \\ Department of Linguistics, Max Planck Institute for Evolutionary Anthropol- \\ ogy, Deutscher Platz 6, D-04103 Leipzig, Germany \& Languages and Cultures \\ of Indian America (TCIA), PO Box 9515, 2300 RA Leiden, The Netherlands. \\ E-mail: wichmann@eva.mpg.de \\ Institute for Theoretical Physics, Cologne University, D-50923 Köln, Eu- \\ roland. E-mail: stauffer@thp.uni-koeln.de \\ Department of Psychology, University of California, Los Angeles, California \\ 9009 5-1563, USA. E-mail: Holman@psych.ucla.edu
}

\begin{abstract}
An earlier study (Nettle 1999b) concluded, based on computer simulations and some inferences from empirical data, that languages will change the more slowly the larger the population gets. We replicate this study using a more complete language model for simulations (the Schulze model combined with a BarabásiAlbert network) and a richer empirical dataset (the World Atlas of Language Structures edited by Haspelmath et al. 2005). Our simulations show either a weak or stronger dependence of language change on population sizes depending on the parameter settings, and empirical data, like some of the simulations, show a weak dependence.
\end{abstract}

Keywords: WALS, computer simulation, diffusion, change rate

\section{Introduction}

Do languages spoken by lots of people change less than those spoken by less people? Common sense says yes: The more people speak a language, the more inertia this language has, similar to the influence of mass on velocity changes in physics. Indeed, one of the successful computer models for language change, the so-called Viviane model (Oliveira et al. 2006, Oliveira et al. 2007) of physicists, assumes this effect from the beginning. However, 
human beings sometimes behave differently from inanimate atoms and thus a direct test of this hypothesis would be desirable.

Nettle (1999b) presents such a test for human languages. He argues that "spreading an innovation over a tribe of 500 people is much easier and takes much less time than spreading one over five million people". His paper mainly contains a computer simulation of language change for just two linguistic features (the simulations can also be interpreted as describing the competition between two languages). He finds that the rate at which the majority of the population switches between these two choices decreases to a small but nonzero limit if the population increases from 120 to 500. Such switching processes could also have been studied in the simpler Ising model of statistical physics, where the switching rate is known (Meyer-Ortmanns and Trappenberg 1990) to decay exponentially to zero with increasing population size. (We also found switching in Nettle's model at high noise level and everybody being influenced by all speakers equally, without having to use any differences in social status, bias, distance or age.) However, these models for only two choices cannot be tested on the empirical language size distribution for the nearly $10^{4}$ human languages, in contrast to the later Viviane, Schulze, and Tuncay language competition models, to be discussed in section 2 .

Our aim is to investigate whether Nettle's result may be replicated if we apply a somewhat different model than the one he described in Nettle (1999a), which was based on the Social Impact Theory of (Nowak et al. 1990). In Nettle's model, the impact of a linguistic variant is a function of the statuses of the individuals using this variant, their social distance from 
the learner, and their number. Our model contains parameters that are similar but not identical. Rather than assigning variable statuses to individuals we operate with a scale-free network, where the impact of a certain individual increases with a probability which is proportional to the impact that the individual already has had. Social distances correspond to distances among individuals in the network which we are using. The size of the population having a given linguistic variant indirectly affects the probability that this variant will diffuse further in one version of our model where a speaker randomly adopts variants from the entire population. The major difference between our model and Nettle's is that ours is more realistic inasmuch as it operates with many languages each of which has several features, whereas Nettle's model, depending on how one interprets it, either has one language with two competing features or two competing languages with no internal structure.

Towards the end of the paper we analyse empirical data and compare our findings with Nettle's inferences based on the empirical data which were available at his time of writing.

\section{Computer Simulation}

\section{$2.1 \quad$ Model}

Of the many computer models for language competition (Abrams and Strogatz 2003, Patriarca and Leppännen 2004, Mira and Paredes 2005, Kosmidis et al. 2005, Pinasco and Romanelli 2006; Schwämmle 2005, see also Cangelosi and Parisi 2002, Culicover and Nowak 2003, Prévost 2003, Itoh and 
Ueda 2004, Wang and Minett 2005), only the Viviane model (Oliveira 2006, 2007), the Tuncay model (2007) and the Schulze model (Schulze et al. 2005, 2007) gave reasonable agreement with the empirical observed distribution of language sizes (where the size of the language is defined as the number of people having this language as their mother tongue.) The Viviane model assumes from the beginning that small languages change more rapidly than large ones. The Tuncay model does not deal with the features of a language and thus seems not suitable to measure language change. The most suitable model for our purposes, then, is the Schulze model, details of which are reviewed in the appendix.

Of the various versions of the Schulze model, we applied the one on scalefree social networks (Barabási and Albert 1999, used for linguistics already by Kalampokis et al. 2007) not only because it gave thus far the best size distribution for languages (Schulze et al. 2007). We also needed it because we wanted to measure change rates. Normally, once a language is spoken by more than half of the people, it keeps that status of dominance forever in the Schulze model. Thus, we observe a situation analogous to what Nettle called the "threshold problem" and described with reference to other scholars before him, such as Keller (1994), who observed that if the learner adopts the norms of his or her immediate surroundings, then the result after a few generations is always "homogeneity if the starting point is heterogeneous and stasis if the starting point is homogeneous" (Keller, 1994: 99, cited after Nettle 1999a: 99). Only on the scale-free networks of Barabási and Albert (1999) did we observe in the Schulze model that the dominating language often changes, as has happened in Europe roughly during the course of the 
last two millennia, where Greek, Latin, French, and English have successively replaced the previous dominating language. In this scale-free network the most connected individuals are responsible for most, if not all, changes in the dominating language. We tested to see what happened if we disallowed modifications in the speech of the centrally connected nodes and found that the dominating language will then not change.

We made $10^{3}$ to $10^{5}$ iterations (sweeps through the network), ignored the first 100 of them, and counted all later changes where the role of the dominating language shifts from one language to another one. We counted both the changes in one arbitrarily selected feature (the first one) and changes in any of the features. If all $F=8$ features would be independent of each other (which they are not) and if all change rates would be small (which they are only in some parameter regions) then the change rates for the first feature would be eight times slower than those for the whole language (denoted by "all" instead of "first", i.e. counting the change in any of the features). Roughly this is the case, i.e. of a pair of curves the higher one counts changes per language, and the lower one counts changes per feature.

\subsection{Local diffusion}

To simulate the process where one speaker adopts a feature from another speaker (diffusion, transfer) we distinguish between a situation where the donor can sit on a neighbour node of the network ("local version") or on any randomly selected node ("global version"). We will start with the local version and then present the global one.

Fig. 1 shows for the local version that neither at intermediate nor at high 


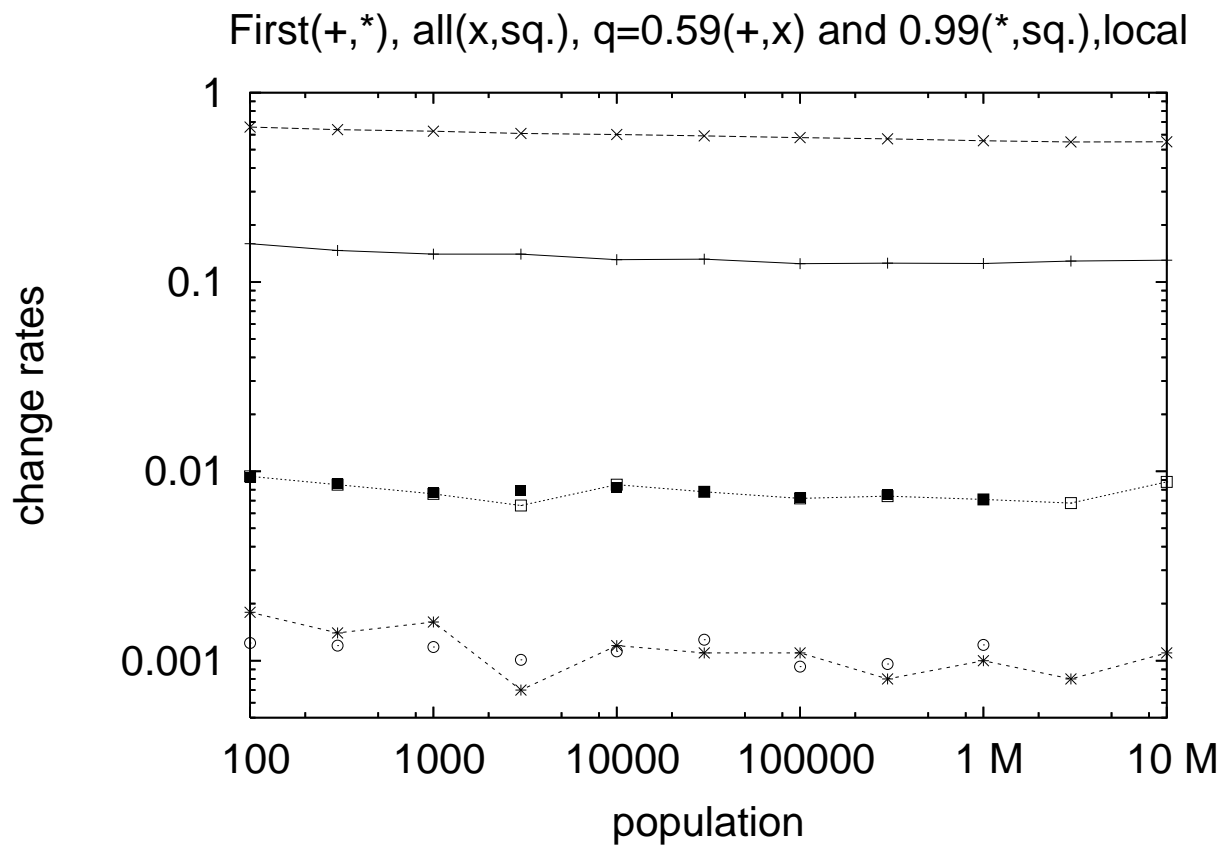

Figure 1: Variation of language and feature change with population size, for intermediate (higher pair of curves) and high (lower pair of curves) diffusion probability. The circles correspond to the stars but with $10^{5}$ instead of $10^{4}$ iterations.

diffusion probabilities $q$ is there a strong variation of change rates with population sizes (= number of network nodes) varying over five decades from 100 to 10 million. However, the stronger coupling between languages at high $q$, compared to low $q$, makes language change more rare. So, these local simulations give a clear answer: Population size has little influence. 


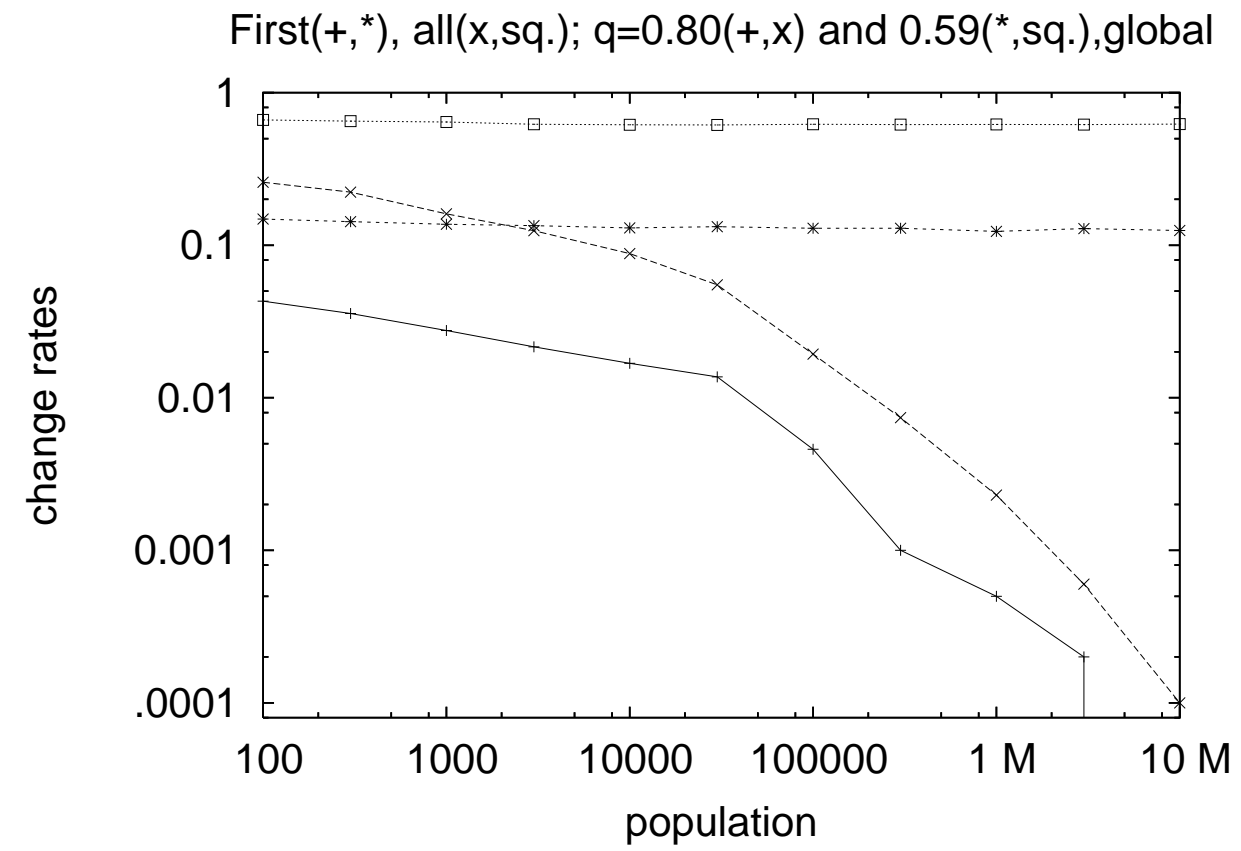

Figure 2: As Fig.1 but now for global instead of local diffusion.

\subsection{Global diffusion}

For global diffusion the situation is quite different. Now for intermediate $q$ again no clear influence of populations size can be seen in Fig. 2, but for larger $q$ the rate of change is diminished drastically with increasing population size. Fig.3 confirms this picture over the whole range of $q$ : Only for large $q$ when the change rates become small does the population size have a strong influence on them. (The analogous figure with local instead of global diffusion has overlapping data, cf. Schulze et al. 2007.) The size effects in the bottom part are in the same direction but stronger than in the top part. (Migration via exchange of nearest neighbours had little influence.)

In the results for both the local and the global situation just reported 

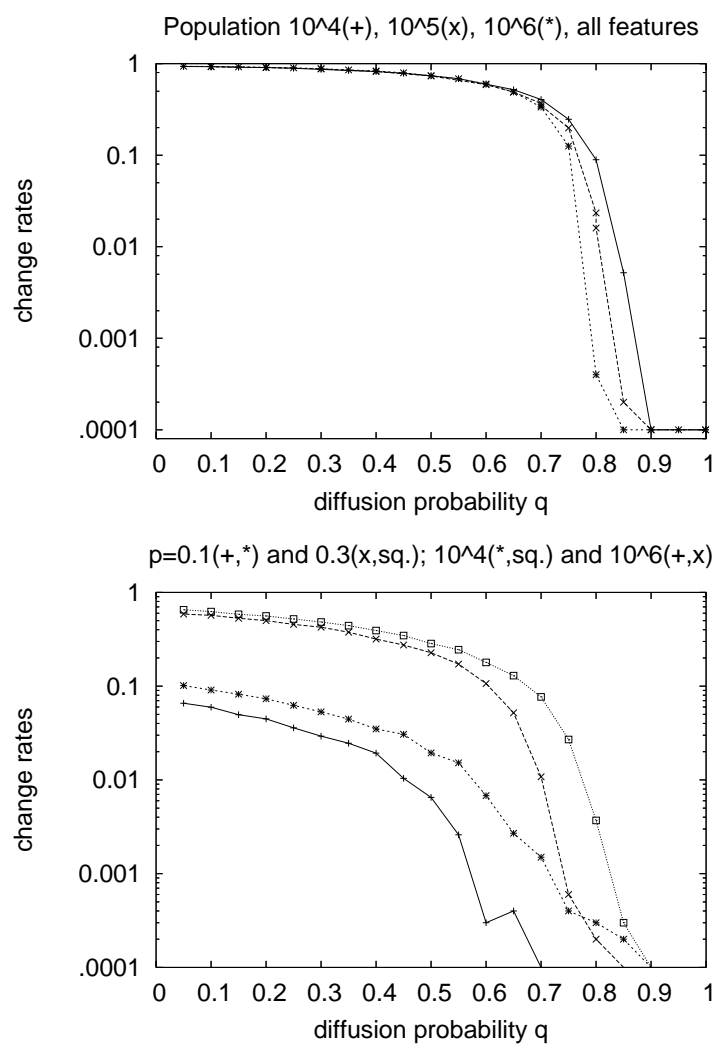

Figure 3: Variation of language change versus diffusion probability: $p=0.5$, 0.3 and 0.1 from top to bottom; population $10^{4} \ldots 10^{6}$.

the complete language shift remains local. If we change from local to global interactions in the shift from one language to the other, then the dominating language always retains its dominating position and no change happens.

One can argue that for small populations global and local diffusion are more similar than for large populations and thus the distinction is less important. Only for large populations does global diffusion give change rates different from their high values for local diffusion. 


\section{Empirical data}

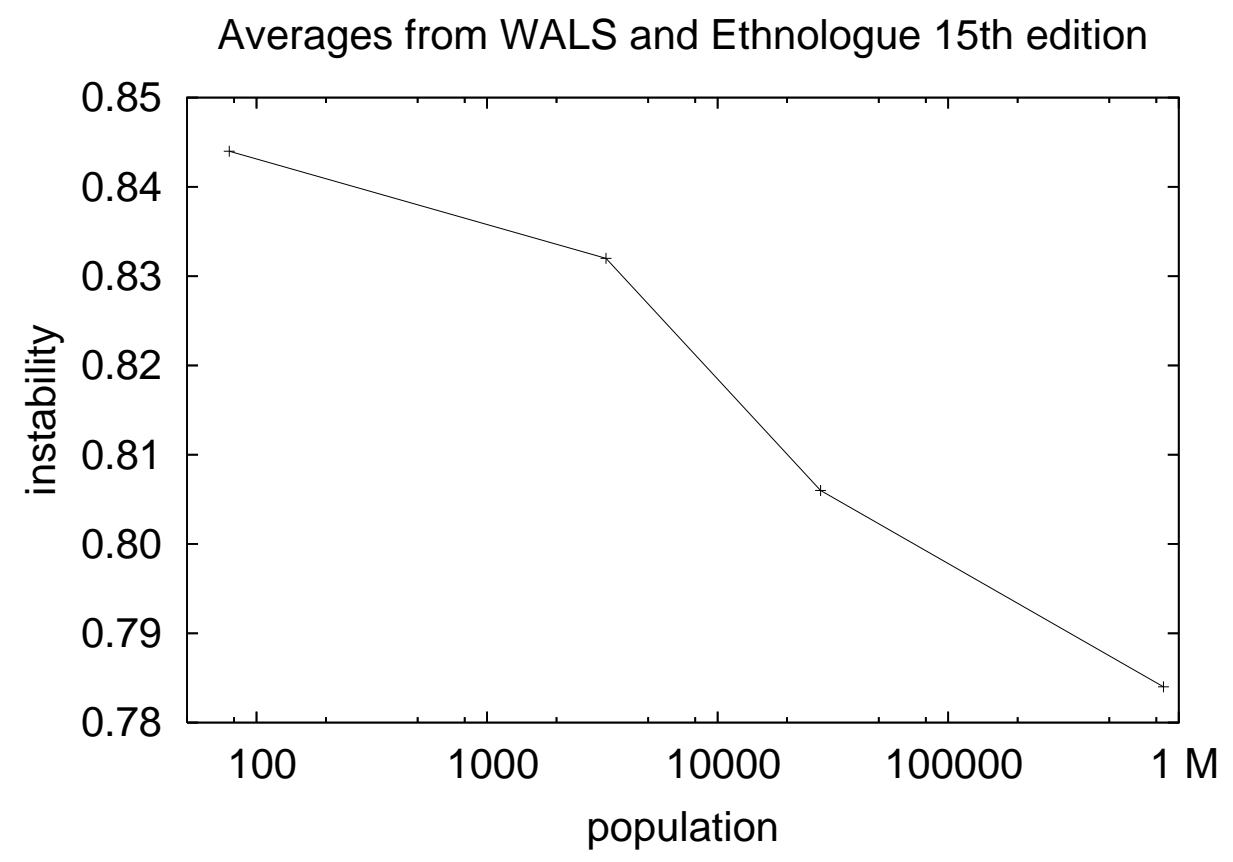

Figure 4: Average instability (= 1 minus stability) versus average population size for real language families.

We used the World Atlas of Language Structures (WALS, Haspelmath et al. 2005), which maps structural features across a number of the world's languages, together with the Ethnologue language statistics (Gordon 2005), to estimate the instabilities and thus indirectly the change rates of real languages.

To study rate of change as a function of number of speakers, the number of speakers of each language was obtained from Ethnologue. Extinct languages and those with unknown numbers of speakers were omitted from the sample. 


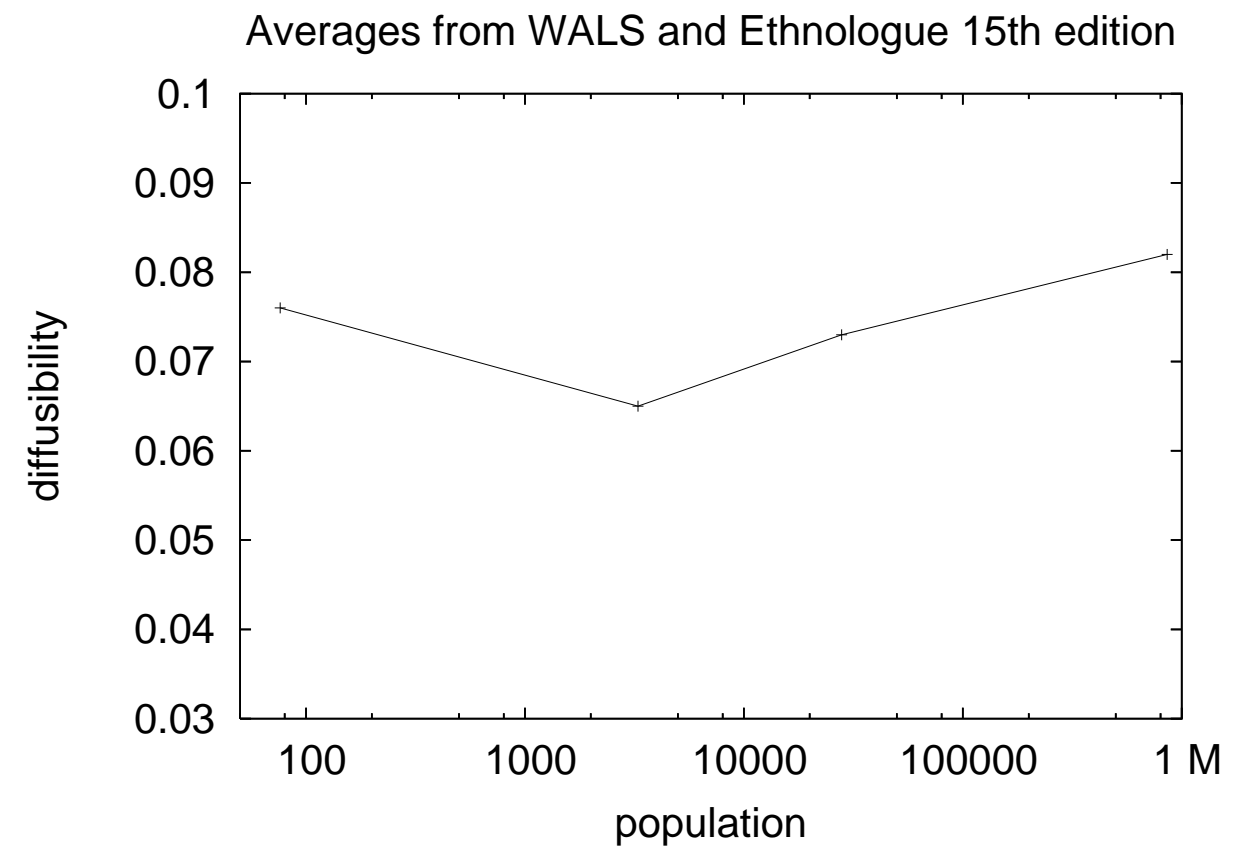

Figure 5: Diffusibility (see text) versus average population size for real language families.

The remaining languages were divided into four groups of approximately equal size: 1 to 999 speakers (423 languages), 1000 to 9999 speakers (513 languages), 10,000 to 99,999 speakers (549 languages), and 100,000 or more speakers (655 languages).

One way to infer rate of change is from the instability of linguistic features by assuming that the more unstable the features are, the faster the rate of change is. Accordingly, the instability of each of the 134 nonredundant WALS features was estimated in each of the four groups of languages. The measure of instability is adapted from Wichmann and Holman (n.d.). (See Holman et al. 2007 for a summary). Within each language family, we look at all the 
pairs of languages located within $5000 \mathrm{~km}$ of each other for which a given feature is attested in both languages, and find the proportion $R$ of such pairs for which the feature has the same value. We do the same for languages in different families, getting the proportion $U$. Then $(1-R) /(1-U)$ is the instability. Instability as defined here is equal to one minus stability as defined in Wichmann and Holman, except that related languages are here considered to be those in the same family rather than those in the same genus, in order to maximize the number of related languages in each group. Thus the values of instability are higher than those inferred from Wichmann and Holman, and the two cannot be compared in absolute terms. Fig. 4 plots the mean stability of the features as a function of the geometric mean number of speakers in the group.

The figure shows a slight decrease in instability with number of speakers. To determine whether the decrease is statistically significant, the Spearman rank correlation between instability and number of speakers was calculated separately for each of the 47 independent features identified by Holman (n.d.). The mean correlation is -0.18 , with a standard deviation of 0.60 ; the mean is significantly negative, $t(46)=2.05, p<0.05$. However, the change is much smaller than in Figs. 2 and 3, and this reality is closer to Fig. 1 based on local diffusion, where also in the population range between 100 and $10^{6}$ a slight decrease of change rates with increasing population was found.

We have also investigated whether diffusibility is dependent on population sizes. Diffusibility is logically independent of stability, since a particular feature may remain stable for a long time after it has diffused. Support for the independence of the two phenomena is provided in Wichmann and Holman 
(n.d.), where it is demonstrated that individual features have comparable stabilities across languages, while the diffusibility of a given feature may vary from area to area.

The diffusibility of each feature was estimated in each of the four groups of languages, again following the procedure described in Wichmann and Holman (n.d.). Among all the pairs of languages in different families located within $5000 \mathrm{~km}$ of each other, we look at the pairs for which a given feature is attested in both languages, and find the proportion $R$ of such pairs for which the feature has the same value. We do the same for pairs of languages in different families located more than $5000 \mathrm{~km}$ each other, getting the proportion $U$. Then $R /(1-U)$ is the diffusibility. Fig. 5 plots the mean diffusibility of the features as a function of the geometric mean number of speakers in the group.

The figure shows little change in diffusibility with number of speakers. The rank correlation between diffusibility and number of speakers has a mean of 0.08 for the 47 independent features, with a standard deviation of 0.66 , indicating no significant correlation, $t(46)=0.84$.

We believe that these empirical findings based on systematic analysis of a large dataset are more solid than the more indirect inferences of Nettle (1999b) and Wichmann (in press) concerning change rates and population sizes. Nevertheless it is interesting that these inferences point in the same direction as the findings based on WALS. Nettle (1999b: 131) observed differences in sizes of languages families in the two hemispheres and explained this by differences in change rates which would in turn be explained by differences in population sizes: 
If languages are changing fast internally, then after they split, identifiable relationship will be quickly erased from their descendants, and so, after a given time period, there will appear to be many, small language families. If the languages are changing very slowly, then identifiable relationship will persist for longer, and so the reconstructable language families will be much broader. In short, a slow rate of change predicts the Old World situation, with few families each of which has many members, whilst a fast rate of change predicts the New World situation, with many families each of which contains few languages.

A modified version of this argument was presented in Wichmann (in press), where not only the number of languages in different families $(n)$ was taken into account, but also the diversity within families $(d)$. For diversity measures glottochronological dates were used. The ratio $n / d$ was labelled the 'density' $(D)$. A correlation was found between small values of $D$ and (present or erstwhile) hunter-gatherer societies and high values of $D$ and sedentary societies whose subsistence has been based on agriculture or fiver fishing. Thus, it was argued, hunter-gatherers tend to live in smaller groups and their languages tend to change faster than sedentary peoples.

We now briefly summarize this section's findings. Population size has no systematic effect on diffusibility. The degree to which languages undergo contact-induced change is probably ultimately dependent on particular histories of interaction among speakers. Internal language change, however, is more constant across language. Nevertheless, our findings show that there is a small but significant effect of language size on the rate of change such that large populations lead to somewhat slower rates of change. More circumstantial empirical evidence discussed in Nettle (1999b) and Wichmann 
(in press) points in the same direction.

\section{Conclusion}

Common sense or analogy with physics and with biology (Oliveira et al. 2006, Sutherland 2003) do not always work: Larger "masses" are not necessarily less mobile, if we identify mass with population size and mobility with language change. We found that only for global as opposed to local diffusion, and for large as opposed to small diffusion probabilities, the rate of language change goes down drastically if the population size increases from 100 to ten million.

We found that only diffusion at the global level may have a size effect. Given a situation where (a) individuals may adopt linguistic features from individuals anywhere in the speech community, (b) certain individuals become more connected than others, and (c) diffusion is high, an increased population size will give a lowered change rate. We can then predict that languages like English or Mandarin Chinese will change more slowly than smaller languages spoken by populations in relative isolation from one another, as we might imagine the situation to have been for some traditional societies. But between these extremes there is a vast gray area of intermediate situations where our simulations hold little predictive power because our parameter values for diffusion, population sizes etc. are abstract and cannot be translated into precise numbers.

Here the empirical data aid us. They indicate that the conditions (ac) mentioned above have never been present to such an extent that, over 
the course of recent millenia, smaller languages have changed much faster than larger ones. Nevertheless, a small but significant effect of population sizes on language change has been observed, supporting the claims of Nettle (1999b), and this should be taken into account when attempts are made to date prehistoric linguistic events.

\section{Appendix}

A language (more precisely, a grammar) in the Schulze model is defined by $F$ features each of which has one of $Q$ values $1,2, \ldots Q$. (We follow Holman et al. 2007 and use $F=8, Q=5$.) It evolves in discrete time steps $t=1,2, \ldots$; all individuals are updated once in each iteration.

All versions of the Schulze model are based on change, diffusion, and shift, using three probabilities $p, q, r$ at each iteration:

- Change: Each feature with probability $p(1-q)$ is randomly changed to a new value between 1 and $Q$.

- Diffusion: Each feature with probability $p q$ is replaced by the corresponding feature from a randomly selected neighbour.

- Shift: Each individual with probability $r(1-x)^{2}$ gives up its language and instead shifts to the language of a randomly selected neighbour.

Here, $x$ for the shift is the fraction of people in the whole population speaking the language of the individual considering a shift. Linguistically these three types of modification may correspond to the analog of biological mutations, to the transfer of linguistic features (loanwords or structural features) from one 
language into another, and to the adoption of a new language, for instance by immigrants.

The simulation first determines, with probability $p$, whether to modify the language, and then does it with probability $q$ by learning from a neighbour and with probability $1-q$ by random change. In our "local" version this neighbour is a nearest neighbour of the considered site, in our "global" version it can be any member of the population. The shift is a conscious decision to give up the own language in favour of a more widespread one.

Usually the individuals sit on sites of a square lattice, but for the present paper they sit on the nodes of a "scale-free" Barabási-Albert network. On these lattices different nodes have different numbers $k$ of neighbours, with a probability proportional to $1 / k^{3}$. These networks are constructed as follows: We start with $m$ nodes having each other as neighbours. Then new members join the network one after the other. They select as their own neighbours (more precisely: teachers) $m$ already existing network members, with a probability proportional to the number of cases where these teachers were selected before by earlier members of the network. Thus popular nodes become even more popular, and unpopular nodes have little chance of becoming selected later. We used these networks instead of square lattices since on lattices the dominating language no longer changes once the majority of people speak it. Mostly we take $m=3$. Our networks are directed, that means if a later node A has selected node B as a teacher ( = neighbour), then B has not selected node $\mathrm{A}$ as a teacher.

We start with everybody selecting randomly one of the $Q^{F}=390625$ possible languages. At each iteration we determine $q_{\max }$ as the most-often 
spoken value of the first feature, and $L_{\max }$ as the most-often spoken language. Then we check how often $q_{\max }$ changes and denote this probability by "first" in some figures. Analogously we count how often $L_{\max }$ changes and mark these probabilities by "all" since all features together determine a language. All changes during the first 100 iterations were ignored. Thus we find the rates at which $q_{\max }$ and $L_{\max }$ change in the stationary regime, while the input parameter $p$ gives the rate at which any feature is modified.

(We also made some tests with $F=1, Q=2$ close to Nettle's model, but then the results were less clear than for our $F=8, Q=5$.)

\section{References}

Abrams, D. and S. H. Strogatz (2003). Modelling the dynamics of language death. Nature 424: 900.

Barabási, L.A. and R. Albert (1999). Emergence of scaling in random networks. Science 286, 509-512.

Cangelosi, A. and D. Parisi (eds.) (2002). Simulating the Evolution of Language. Berlin: Springer-Verlag.

Culicover, P. and A. Nowak (2003). Dynamical Grammar, Oxford University Press, Oxford

Gordon, R. G., Jr. (ed.). (2005). Ethnologue: Languages of the World, Fifteenth edition. Dallas, Tex.: SIL International. Online version: http://www.ethnologue.com/.

Haspelmath, M., M. Dryer, D. Gil, and B. Comrie (eds.) (2005). The World Atlas of Language Structures. Oxford: Oxford University Press. 
Holman, E. W. (no date). Approximately independent typological features of languages. Submitted for publication.

Holman, E. W., C. Schulze, D. Stauffer, and S. Wichmann (2007). On the relation between structural diversity and geographical distance among languages: observations and computer simulations. Conditionally accepted by Linguistic Typology. Preprint available at http://email.eva.mpg.de/ wichmann/HolmanRevisedSu Itoh, Y. and S. Ueda (2004). The Ising model for changes in word ordering rules in natural languages. Physica D 198: 333-339.

Kalampokis A., K. Kosmidis, and P. Argyrakis (2007). Evolution of vocabulary on scale-free and random networks. Physica A 379: 665-671.

Keller, R. (1994). On Language Change: The Invisible Hand in Language. London: Routledge.

Kosmidis, K., J.M. Halley, and P. Argyrakis (2005). Language evolution and population dynamics in a system of two interacting species. Physica A 353: 595-612.

Meyer-Ortmanns, H. and T. Trappenberg (1990). Surface tension from finitevolume vacuum tunneling in the 3D Ising model. Journal of Statistical Physics 58: 185-198.

Mira, J. and A. Paredes (2005). Interlinguistic simulation and language death dynamics. Europhysics Letters 69.6: 1031-1034.

Nettle, D. (1999a). Using social impact theory to simulate language change. Lingua 108: 95-117.

Nettle, D. (1999b). Is the rate of linguistic change constant? Lingua 108: 119-136. 
Nowak, A., J. Szamrej, and B. Latane (1990). From private attitude to public opinion: A dynamical theory of social impact. Psychological Review 97, 362-376.

Oliveira, V. M. de, M. A. F. Gomes, and I. R. Tsang (2006). Theoretical model for the evolution of linguistic diversity. Physica A 361: 361-370.

Oliveira, P. M. C. de, D. Stauffer, F. S. W. Lima, A. O. Sousa, C. Schulze, and S. Moss de Oliveira (2007). Bit-strings and other modifications of Viviane model for language competition. Physica A 376, 609-616.

Patriarca, M., T. Leppännen (2004). Modeling language competition. Physica A 338: 296-299.

Pinasco, J. P., L. Romanelli (2006). Coexistence of languages is possible. Physica A 361: 355-360.

Prévost, N. (2003). textitThe Physics of Language: Towards a Phase Transition of Language Change. PhD dissertation, Simon Fraser University.

Schulze, C. and D. Stauffer (2005). Monte Carlo simulation of the rise and fall of languages. International Journal of Modern Physics C 16: 781-787.

Schulze, C., D. Stauffer, and S. Wichmann (2007). Birth, survival and death of languages by Monte Carlo simulation. Conditionally accepted by Communications in Computational Physics,.

Schwämmle, V. (2005). Simulation for competition of languages with an ageing sexual population. International Journal of Modern Physics C 16: $1519-1526$.

Sutherland, W. J. (2003). Parallel extinction risk and global distribution of languages and species. Nature 423: 276-279. 
Tuncay, Ç. (2007). Physics of randomness and regularities for cities, languages, and their lifetimes and family trees. International Journal of Modern Physics C 18, in press.

Wang, W. S. Y., Minett J. W. (2005). The invasion of language: emergence, change and death. Trends in Ecology and Evolution 20.5: 263-296.

Wichmann, S. In press. Neolithic linguistics. In: Barjamovic, G., I. Elmerot, A. Hyllested, B. Nielsen, and B. Okholm Skaarup (eds.), Language and Prehistory of the Indo-European peoples - A Cross-Disciplinary Perspective. Budapest: Archaeolingua.

Wichmann, S. and E.W. Holman (no date). Assessing temporal stability for linguistic typological features. Submitted. Preprint available at http://email.eva.mpg.de/ wichmann/WichmannHolmanIniSubmit.pdf. 